\title{
Le rapport entre éthique et politique : un enjeu pour l'éducation relative à l'environnement
}

Lucie Sauvé

\section{(2) OpenEdition}

Journals

Édition électronique

URL : http://journals.openedition.org/ere/2229

DOI : 10.4000/ere.2229

ISSN : 2561-2271

Éditeur

Centr'ERE

Référence électronique

Lucie Sauvé, « Le rapport entre éthique et politique : un enjeu pour l'éducation relative à

l'environnement », Éducation relative à l'environnement [En ligne], Volume 8 | 2009, mis en ligne le 20

décembre 2009, consulté le 21 février 2020. URL : http://journals.openedition.org/ere/2229; DOI

10.4000/ere.2229

Ce document a été généré automatiquement le 21 février 2020. 


\title{
Le rapport entre éthique et politique : un enjeu pour l'éducation relative à l'environnement
}

\author{
Lucie Sauvé
}

1 On le reconnaît aisément, l'éducation et l'environnement sont deux mondes chargés de valeurs. Deux champs «minés» de valeurs peut-on dire, si l'on fait référence aux risques que peuvent générer des choix éthiques non clarifiés. Pas étonnant que lorsqu'on conjugue éducation et environnement pour aborder le rapport à l'environnement, on retrouve un bouillon de valeurs particulièrement effervescent. Au cœur de l'action éducative, souvent liée à l'action sociale, cela fait appel à une grande exigence de lucidité, de rigueur, d'authenticité et d'intégrité. D'autant plus que les choix éthiques en matière d'environnement ont une composante politique, impliquant une dynamique et des répercussions collectives: l'environnement est une "affaire publique », c'est un milieu de vie partagé, incluant entre autres, un ensemble de ressources communes. Mais cette dimension politique est également associée au processus ontogénique de la construction d'une identité environnementale, tant individuelle que sociale : elle suppose la construction d'une «identité politique ». L'un des objectifs de l'éducation relative à l'environnement est précisément de stimuler et d'accompagner ce processus identitaire, indispensable à la reconstruction du rapport à l'environnement.

2 En premier lieu, cet article traite des principales questions qui émergent lorsqu'on aborde en éducation l'idée de "valeurs environnementales » et d'éthique. Puis, nous explorerons sommairement la diversité des propositions relatives au champ de l'éthique de l'environnement et mettrons en lumière la dimension politique de cellesci. Enfin, la démarche et certains résultats d'une recherche portant sur les initiatives et les acteurs d'une éducation relative à l'éco-alimentation permettront d'illustrer les éléments théoriques esquissés dans cet article. À partir de cet exemple, l'idée d'engagement apparaît comme l'une des charnières possibles entre l'éthique et le politique. 


\section{Un certain détour du côté de l'amour}

En ce qui concerne les valeurs du monde de l'éducation, le récent ouvrage de Daniel Pennac (2007), Chagrins d'école, est particulièrement éclairant. L'auteur présente un récit autobiographique de sa propre trajectoire d'élève (un "cancre») et puis d'enseignant préoccupé de rejoindre les jeunes en difficulté d'apprentissage. L'ouvrage est traversé par un regard critique sur l'éducation et plus spécifiquement par une implacable introspection de praticien réflexif. La grande question du "pourquoi » est associée à celle du «comment» éduquer. Le livre se termine avec l'idée d'amour : l'amour comme fondement et condition de l'acte éducatif.

4 Il ne s'agit pas d'un amour naïf et mièvre, mais d'un amour lié à l'exigence pour chacun de s'engager dans la tâche de vivre et d'apprendre (les deux étant si liés), un amour de l'autre (l'élève) qui renforce la confiance en soi et l'espoir qu'on peut «y » arriver. À travers l'ensemble de l'ouvrage, c'est précisément ce «y » qui est examiné : à quoi veuton arriver au juste et pourquoi? L'auteur témoigne d'un exercice constant de clarification des objectifs, des valeurs, des désirs, des peurs, des obstacles à franchir ... tant pour lui-même que pour les jeunes. Pour ceux qui adhèrent au courant humaniste de l'éducation, cela peut paraître normal, voire banal, qu'un texte sur l'éducation traite d'amour. Ce qui est neuf dans l'ouvrage de Pennac, c'est l'argumentaire de l'auteur pour « $\mathrm{y}$ » arriver.

5 Et en matière d'environnement, est-il possible de considérer également l'amour comme une valeur de base? Quand on dit qu'il faut "aimer l'environnement ", on prend en réalité un drôle de raccourci... Si l'on pense, par exemple, à certains quartiers défavorisés de nos villes, à nos univers bétonnés, commercialisés, artificialisés, aux régions inondées où les récoltes sont détruites et où prolifèrent les insectes porteurs de malaria, on ne peut pas « aimer » ces environnements-là. Il faut plutôt dénoncer, lutter, réparer, déconstruire, reconstruire... Alors quand on dit qu'il faut «aimer l'environnement ", quand on affirme que l'un des objectifs de l'éducation relative à l'environnement est de "transmettre l'amour de l'environnement ", à quoi fait-on référence? Quelles sont ces fameuses «valeurs environnementales» qu'on souhaite « inculquer»? Et faut-il «inculquer» des valeurs, si vertueuses soient-elles à nos yeux?

\section{« II n'existe pas de valeurs environnementales »}

6 Ces questions me rappellent un incident critique... Un étudiant de doctorat préparait une thèse portant sur «l'éducation relative aux valeurs environnementales"; il s'intéressait aux théories et pratiques des enseignants du primaire à cet effet. Lorsqu'il présenta son projet de recherche au comité chargé de le valider, un évaluateur lança un énorme pavé dans la mare : «Vous faites preuve d'un grand enthousiasme et annoncez une méthodologie rigoureuse, mais à propos de quoi au juste? Votre recherche n'a pas d'objet, puisqu'il n'existe pas de valeurs environnementales ». Après un lourd moment de malaise au cours d'une trop brève discussion tendue et maladroite de part et d'autre, l'étudiant rebondit et promit de relever le défi de reconstruire un cadre théorique 
permettant d'aborder un tel postulat dans une perspective critique. L'exercice en valut la peine.

Depuis ce temps, chaque année, les étudiants de notre programme de formation en éducation relative à l'environnement sont invités à clarifier les fameuses «valeurs environnementales $»^{1}$. L'exercice débute par un moment de silence où chacun(e) est invité(e) à dresser une liste de ces valeurs. Au début, les crayons s'agitent : ... respect, responsabilité, solidarité... Et puis, les minutes paraissent longues... Lors de l'échange qui suit, on se rend vite compte que les valeurs exprimées sont en réalité d'ordre instrumental: Respect de quoi au juste? Pourquoi? À quelle fin? Respect de l'environnement, précise-t-on. Mais qu'entend-on par environnement? Un ensemble de ressources à ne pas épuiser pour assurer la durabilité du développement? La nature, porteuse d'une valeur intrinsèque? Qu'inclut-on dans l'idée de nature? On creuse encore davantage : Respect de la vie - de la Vie. Mais alors de quelle vie ? Celle qui monte dans la sève des arbres centenaires de forêts sacrées ne pose pas de problème. Mais qu'en est-il de la vie de l'agneau dont on mange les côtelettes? Et de celle de l'insecte piqueur? Et de celle du loup qu'on veut réintroduire en montagne? La discussion s'anime et pourrait bien s'éterniser... On se rend compte que le sens de la vie n'est pas le même pour tous les groupes sociaux, dans toutes les cultures. Ainsi, pour les tenants du mouvement Pro-vie contre l'avortement, l'idée de vie n'a certes pas la même connotation que pour les militants de Greenpeace contre les organismes génétiquement modifiés (OGM). Le monde des valeurs est plein de «mots valises » qui réservent des surprises à la fouille...

8 Cette discussion collective permet d'explorer la bipolarité du domaine des valeurs: d'une part, on retrouve des objets porteurs de valeur (comme les espèces menacées) et d'autre part, des valeurs qui sont en fait des "attitudes" envers ces objets (par exemple, la sollicitude ou le care); aussi, les valeurs instrumentales (comme la démocratie ou la "soutenabilité») se distinguent des valeurs fondamentales, individuelles ou collectives, au regard de l'environnement, comme le bien-être des populations humaines ou l'intégrité des systèmes de vie; les valeurs intrinsèques se distinguent également des valeurs extrinsèques: ainsi, la biodiversité peut être célébrée pour elle-même, mais elle est le plus souvent valorisée comme ressource à exploiter. La discussion permet également de remettre en cause l'existence de « vraies valeurs", de "valeurs universelles ». Les valeurs sont socialement construites et culturellement déterminées. On prend conscience qu'une valeur n'a de sens que dans le système de valeurs où elle s'insère. D'où l'intérêt de dresser une carte en réseau de ses valeurs " personnelles ", de les expliciter, de les justifier, les comparer, les confronter... de saisir qu'elles sont issues d'interactions sociales au sein des divers groupes d'appartenance qui se sont succédés ou superposés au fil de nos vies, et que le système qu'elles forment est plus ou moins cohérent, qu'il comporte des interstices, des vides, des points aveugles, des ruptures... On ne met pas aisément les valeurs en petites boîtes!

Et puis on se rend compte qu'il faut distinguer la morale et l'éthique. Pas si facile... Les auteurs ne s'accordent pas tous sur la définition de l'une et de l'autre et sur la relation entre les deux. Mais de façon générale, on peut convenir que la morale serait un ensemble de valeurs données (bien qu'évolutives) au sein d'un groupe social, explicitement ou implicitement prescrites, qui vise l'adoption d'un code de comportements. Quant à l'éthique, elle correspond au processus et au résultat d'une 
démarche de construction d'un système de valeurs, sans cesse confrontées à la mise en acte dans divers contextes. Morale et éthique apparaissent comme deux champs distincts dont il reste à clarifier les passerelles : d'un côté, un code de comportements versus un processus menant à des conduites, à un agir délibéré ; la morale comme objet de prescription versus un projet éthique; une logique de "transmission » versus une logique de construction individuelle et collective; une activité d'enseignement des valeurs versus une démarche d'éducation, menant au développement d'une compétence éthique. Une telle compétence (CSE, 1990) suppose l'acquisition d'une certaine culture du domaine de l'éthique (la connaissance de diverses postures éthiques), un savoir-faire en matière d'analyse critique et de prise de décision, et un savoir-être (exigence de rigueur par exemple ou d'authenticité) en vue de pouvoir adopter un agir éthique cohérent et approprié au regard du contexte.

Mais si la morale et l'éthique sont généralement associées à la vertu, il importe également de mettre au jour les limites et dérives possibles de l'argument moral, de même que les perversions associées à certaines postures éthiques. Le discours de la morale ou de l'éthique ne sert-il pas trop souvent d'argument pour implanter ou maintenir un certain pouvoir politique, religieux, économique, etc.? Ainsi, la " démocratie » n'est-elle pas devenue une puissante arme de guerre pour justifier par exemple l'invasion barbare de pays "sous-développés ", riches en pétrole ? Également, comme le montre Marie-Monique Robin (2008), les valeurs prônées par la firme Monsanto - dialogue, transparence, respect, partage, utilité - prennent un sens particulier et paradoxal lorsqu'elles sont examinées à la lumière des finalités de l'entreprise et des actions qu'elle engage.

\section{Qu'en est-il plus spécifiquement de l'éthique de l'environnement?}

11 Sans doute convient-il mieux de parler du « champ » de l'éthique de l'environnement (champ social - au sens donné par Pierre Bourdieu - où se déploie une pluralité de théories et pratiques) plutôt que d'une éthique de l'environnement, au singulier. Il existe en effet une grande diversité de propositions éthiques, chacune témoignant d'une certaine vision de l'environnement et mettant de l'avant certaines valeurs (voir le tableau 1). Les valeurs privilégiées (souvent les mêmes) prennent leur sens particulier au sein du système de valeurs où elles s'insèrent, en fonction d'une certaine façon d'envisager le rapport à l'environnement. 
Tableau 1 : Diversité de propositions éthiques en matière d'environnement : quelques éléments de caractérisation (d'après, entre autres, Desjardins, 1995 ; Larrère, 1997 ; Sauvé et Villemagne, 2003)

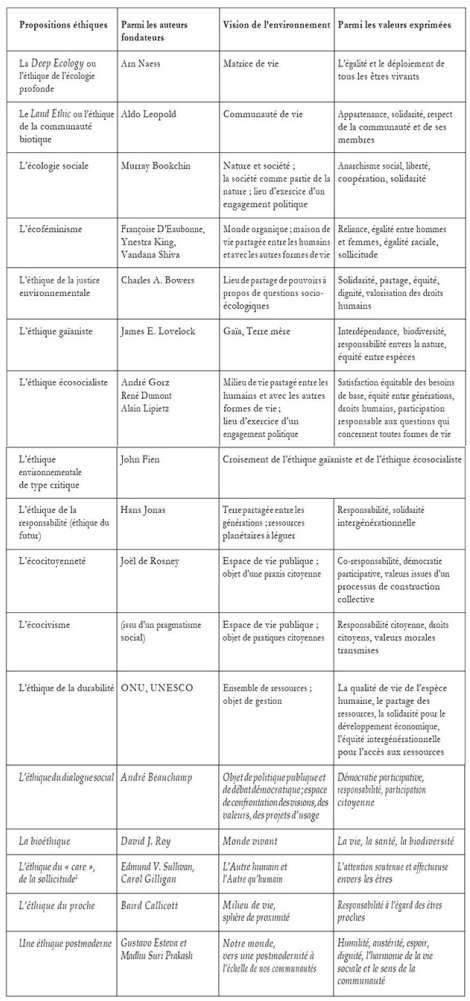

NOTE : Les propositions éthiques en italique ne sont pas spécifiques ou nécessairement explicites à l'égard du rapport à l'environnement.

Certes, ce tableau reste à compléter. D'autres propositions pourraient y être considérées. Par exemple, cette éthique de l'amour (comme en éducation!) qui a été esquissée en conclusion du diagnostic des effets de la croissance économique sur l'environnement planétaire, réalisé par Donella et Denis Meadows et Jorgen Randers (2004, p. 281-284). Par ailleurs, on peut se demander si une éthique de la " soutenabilité » (ou viabilité) est en voie de définir ses fondements et caractéristiques propres (s'il en est), distincts de ceux des propositions antérieures du champ de l'éthique de l'environnement et distincts également des rudiments d'une éthique du développement durable telle que formulés par les instances internationales qui en font la promotion.

\section{La dimension politique de l'éthique de l'environnement}

Plusieurs propositions éthiques mentionnées au tableau 1 font référence à la dimension politique du rapport à l'environnement. Elles nous invitent à nous pencher sur l'environnement comme «chose publique» (donc politique), comme objet de préoccupations sociales. Les innombrables controverses à propos des questions environnementales (comme les usages de l'eau ou l'aménagement du territoire) nous ont amenés à reconnaître à l'évidence que l'environnement correspond à un ensemble de réalités de nature socio-écologique et mettent en relief la dimension sociale, collective et politique de l'environnement. 

collective, est sans cesse à construire et reconstruire, ici, entre nous, à propos de nos «affaires » communes, à propos d'une problématique particulière qui nous confronte, au creux de nos cultures de référence qu'il importe également de clarifier. Chaque situation environnementale est unique, évolutive. Au-delà des lieux communs, des « valeurs de base », cela fait appel à une clarification des fondements et visées de nos prises de position individuelles et collectives et de ce que cela implique pour la mise en actes concrets de ces dernières. Certes, les diverses propositions éthiques (comme celles qui sont identifiées au tableau 1) sont de nature à éclairer et enrichir la démarche éthique. D'où l'intérêt de mettre en œuvre une éducation relative à l'environnement qui favorise le développement d'une culture éthique, comme composante d'une compétence éthique.
L'éthique de l'environnement est à faire [...] J'aurais peur pour ma part d'une éthique en vase clos, de l'élaboration théorique par quelque savant, expert, écologue ou militant, d'un schéma abstrait idéal [...] À mes yeux, l'antidote à une éthique close, rigoureuse, étouffante à la limite, c'est l'opinion publique [...] qui surgit des consultations publiques longuement et patiemment élaborées où les informations ont pu être vérifiées et scrutées en public, où les valeurs proposées, explicites et implicites, ont été discutées au mérite [...]. (André Beauchamp, 1991, p. 141)

L'éthicien André Beauchamp fait ici référence à l'intelligence collective quand elle devient une intelligence citoyenne ${ }^{2}$, affinée par le dialogue social. Il puise à même sa riche expérience d'enquête à travers les consultations du Bureau des audiences publiques en environnement au Québec (BAPE).

Parmi les controverses qui ont fait l'objet de telles consultations, celle du développement de l'industrie porcine ${ }^{3}$ offre un bon exemple des défis associés à l'émergence d'une intelligence citoyenne sur une question socio-écologique. On retrouve d'une part, la volonté de l'État de soutenir et développer une telle industrie comme fer de lance de l'économie agricole (malgré les aléas de la mondialisation et des épidémies virales au sein des troupeaux); d'autre part se manifestent les multiples formes d'opposition citoyenne évoquant principalement les problèmes écologiques, sanitaires, sociaux et économiques que soulève un tel projet et réclamant l'appui politique au développement d'une agriculture alternative. Entre les deux, diverses voix se font entendre chez les agriculteurs: le lobby des promoteurs industriels, les revendications des éleveurs qui souhaitent une plus grande reconnaissance symbolique et financière de leur travail, le désespoir de ceux qui font faillite ou qui sombrent en dépression à cause de l'ampleur de leur tâche et des inquiétudes financières, le désir de reconnaissance de ceux qui font preuve d'innovation en matière agro-alimentaire, etc.

18 L'analyse des rapports de la consultation publique (BAPE, 2003) permet de mettre en évidence, au premier plan, des enjeux de savoirs. Comme pour toutes les questions de ce type, une tension peut être aisément observée entre les savoirs légitimés, 
socialement valorisés (d'ordre techno-scientifique), et les savoirs d'expérience (d'ordre empirique), vus comme divergents ou opposés plutôt que complémentaires. On se rend vite compte que l'information est le nerf de la guerre, au cœur de rapports de pouvoir. or la question est si complexe... Certains acteurs (fonctionnaires ou scientifiques) manipulent bien le langage souvent mystificateur des variables et des chiffres. D'autres tentent avec conviction, parfois maladroitement, de braquer le projecteur sur les réalités, problèmes ou risques observés, ressentis ou pressentis, dont la nature qualitative rend difficile la démonstration.

Mais l'analyse du dossier de cette consultation publique permet également de mettre en lumière des enjeux éthiques ${ }^{4}$. Les valeurs sont rarement clarifiées par les protagonistes. Or on observe que c'est justement sur le plan des valeurs que sont souvent dénigrés les arguments de ceux qu'on appelle avec une certaine dérision «les citadins » (soit les ruraux ou néo-ruraux dont le travail n'est pas lié à l'agriculture et qui s'opposent à l'élevage intensif du porc). Les promoteurs les disent mal informés de la condition agricole, mais surtout « émotifs », donc pas crédibles. On pointe du doigt la valeur égoïste du "pas dans ma cour ", motivation occulte dit-on, à la base de leurs revendications liées à la seule valeur immobilière de leur propriété et à leur crainte non fondée de perte de qualité de vie individuelle (s'opposant ainsi au développement économique collectif et à l'approvisionnement alimentaire de la population). Il est vrai qu'un tel argument, bien que légitime, n'est pas suffisant pour rejoindre le plan politique. Comment montrer que la réaction première du " pas dans ma cour » (et on se rend compte que la «cour " s'étend finalement à la dimension de nombreux bassins versants) est un déclencheur pour une réflexion en profondeur sur notre rapport à la terre, aux animaux, à l'intégrité de la vie (si l'on pense aux manipulations génétiques), à la santé des écosystèmes et des populations, à l'alimentation, au travail agricole, au développement régional, à la solidarité, etc. ? Les protagonistes d'une telle question socio-écologique auraient tout avantage à construire leur propre argumentaire et aussi à déconstruire si nécessaire celui des autres, non seulement sur le plan des savoirs, mais aussi sur celui des valeurs.

Cela interpelle une éducation relative à l'environnement en milieu communautaire (à l'échelle du quartier, du village, des regroupements de citoyens, etc.), de nature à mettre en évidence les extraordinaires possibilités de l'apprentissage dans l'action sociale, qu'il s'agisse d'une action de résistance (dans la dénonciation ou le refus) ou d'une action créative, à travers le développement de projets collectifs. Dans un tel contexte, l'« éducation » devient animation, comme c'est souvent le cas en éducation populaire où l'animation socioculturelle devient un axe de travail central. D'où l'intérêt de former des leaders communautaires (qu'ils soient élus, mandatés ou «naturels») capables de stimuler, d'accompagner et d'éclairer une dynamique citoyenne autour d'un enjeu collectif. Or précisément, l'un des éléments de formation des leaders en matière d'environnement (ou d'écoleaders) est celui de reconnaître l'importance de prendre en compte l'identité écologique des gens, de clarifier cette identité, de renforcer ou d'enrichir s'il y a lieu cet aspect de l'identité individuelle et collective qui concerne le rapport au monde, à Oikos, cette maison partagée entre nous les humains et les autres vivants. Une telle identité comporte une dimension politique, observe Mitchel Thomashow (1995). Celle-ci, dit-il, est liée à la vision de son propre pouvoir face aux situations, aux autres, aux institutions. Quelles sont mes (nos) options politiques? Quelles sont mes (nos) réactions face aux enjeux de pouvoir, face aux situations de conflits sociaux par exemple? Quel est mon « tempérament » politique? Qu'en est-il de 
notre «culture » politique? Quelles sont mes (nos) actions politiques en ce moment? Sont-elles délibérées ou non? Sont-elles pro-actives ou passives? Quelles actions politiques pourrais-je (pourrions- nous) entreprendre ? Dans quel but?

\section{L'alimentation, entre éthique et politique}

21 À la Chaire de recherche du Canada en éducation relative à l'environnement ${ }^{5} \mathrm{de}$ l'Université du Québec à Montréal, nous nous intéressons à cette dimension politique du rapport au monde. En particulier, nous menons actuellement une recherche sur l'éducation relative à l'alimentation, dans une perspective de santé environnementale. L'alimentation se retrouve en effet au cœur de notre rapport à l'environnement. À travers les aliments, nous mangeons littéralement notre environnement; ou encore, nous mangeons des environnements lointains à travers les denrées d'importation. Nous «mangeons » l'eau, la pluie, les molécules de l'air, les minéraux du sol et l'énergie du soleil qui ont fabriqué les aliments, mais nous ingérons aussi les pesticides et autres ingrédients anthropiques. Pour le meilleur et pour le pire, notre santé est étroitement liée à celle des écosystèmes. Également, nos choix alimentaires portent un message : quand j'achète et consomme tel ou tel type d'aliment, j'encourage un certain système de production et de distribution. Manger devient un acte à dimension éthique et politique. C'est dans cette perspective que nous avons entrepris une recherche sur les théories et pratiques de l'éducation relative à l'éco-alimentation en milieu non formel (organisations non gouvernementales, fermes, coopératives, restauration, etc.). L'écoalimentation correspond à une alimentation saine (diversifiée, sécuritaire), produite, distribuée et consommée dans le respect des processus écologiques et de l'équité des rapports sociaux.

Dans un premier temps, nous avons repéré plus de 80 initiatives d'éducation en ce sens au Québec. Nous souhaitons valoriser ces initiatives, faire une synthèse critique des théories et pratiques qui s'y déploient, en vue de contribuer à constituer le champ de l'éducation relative à l'éco-alimentation ${ }^{6}$. Plus spécifiquement, nous sous intéressons à l'argumentaire des acteurs de ces initiatives et aux valeurs associées à cet argumentaire.

Entre autres, nous pouvons globalement observer qu'il existe à travers les discours, un spectre d'arguments éthiques associés à certains registres de valeurs :

- Égocentrisme : on y trouve l'engouement pour le «manger bio » et les « alicaments ", issu généralement d'une préoccupation de santé personnelle : éviter les maladies (en particulier les maladies cardiovasculaires et le cancer) et/ou être en meilleure forme physique et mentale ;

- Sociocentrisme : on se préoccupe ici de la sécurité alimentaire dans une perspective de santé publique; la traçabilité devient entre autres une préoccupation pour s'assurer que les aliments soient sains, exempts de risques. On met en évidence l'importance du partage équitable de ressources alimentaires de qualité : le bio n'est pas que pour les riches! On prône la souveraineté alimentaire en vue d'échapper à l'aliénation de la globalisation ; on favorise l'économie locale, biorégionale. À travers les initiatives comme celle du commerce équitable, l'idée de solidarité s'élargit à l'échelle globale ;

- Biocentrisme : au centre du discours, on retrouve le respect de la vie, celle des animaux entre autres, dont on dénonce les conditions d'élevage. La lutte contre les OGM s'inscrit dans une dénonciation de l'ensemble des manipulations génétiques à visées productivistes. On 
valorise la biodiversité, pour sa valeur intrinsèque. Manger nous met en communion avec le vivant ;

- Écocentrisme : on met l'accent sur les liens entre la santé humaine et celle des écosystèmes. La logique écosystémique permet de relier l'écologique et le social, le local et le global : le jardin s'inscrit dans un bassin versant, puis il devient une parcelle du grand jardin planétaire; il s'agit là de diverses dimensions d'un même Oikos. Manger nous inscrit dans une dynamique écosystémique, comme partie prenante des flux et cycles, partageant une responsabilité globale.

24

\section{L'engagement : un lien entre éthique et politique?}

5 L'enquête que nous avons menée auprès de nombreux acteurs des organisations porteuses d'initiatives en éducation relative à l'éco-alimentation met en évidence les valeurs de l'ancrage et de l'engagement dans le milieu de vie. Le localisme et le biorégionalisme apparaissent comme des références de base. L'idée de solidarité sociale ressort également. Mais, qui donc sont ces gens qui s'engagent en éco-alimentation? Pourquoi le font-ils ? À quoi la notion d'engagement fait-elle référence?

Jean Ladrière (sans date) a mis en évidence le caractère identitaire de l'engagement et sa dimension ontogénique. S’engager implique de se définir, de choisir une avenue d'être au monde, de donner un sens à son agir et de se projeter dans l'avenir : il s'agit d'un acte identitaire. Mais au-delà d'un acte, l'engagement est aussi une conduite, observe Ladrière: cela correspond à un style d'existence qui consiste à assumer personnellement et activement une situation, à associer sa propre existence à celle des autres et au fil d'un projet qu'on assume. L'engagement est contraire à la fatalité ; il implique de croire qu'on peut contribuer à changer les choses, qu'il y a une marge de manœuvre, un espace de liberté à saisir. On retrouve ici une caractéristique constante des acteurs que nous avons interrogés, qui par ailleurs proviennent de diverses sphères de formation et d'expérience. Enfin, parce qu'il met en projet, l'engagement devient anticipateur et émancipateur. Il comporte un potentiel d'actualisation et de transformation.

En lien avec sa dimension ontogénique, l'engagement comporte certes également une dimension éthique. Si l'engagement peut se réaliser dans la sphère personnelle et individuelle (l'engagement artistique ou spirituel par exemple), le plus souvent, il fait référence à un acte social, comme un acte de responsabilité par excellence, responsabilité d'être et d'agir en cohérence, ce qui suppose la clarification de ses valeurs fondamentales. On retrouve ainsi avec l'éthique, la dimension politique de l'engagement, en lien et au-delà d'un « engagement politique » éclairé. À cet effet, Paul Ricoeur (1986) déplore qu'en politique, les valeurs sont généralement amputées de leurs racines. Elles sont figées dans des stéréotypes. On n'ose pas les déterrer afin de préserver une certaine paix sociale, parce qu'au fond, on le sait bien, les motivations profondes sont multiples et conflictuelles. Les valeurs sont comme des fleurs coupées dans un vase observe Paul Ricoeur (1986, p. 405) : sans racine, elles sont une nature morte. D'où l'importance d'un engagement citoyen critique, attentif au lien entre éthique et politique. 
D'une part, notre exploration de la problématique agroalimentaire a bien mis en lumière l'utilisation abusive du discours éthique pour justifier les choix politiques dans les sphères de décision politico-économiques. Mais d'autre part, notre enquête auprès des acteurs de l'éco-alimentation, en particulier de l'éducation relative à l'écoalimentation, nous amène au cœur d'un engagement citoyen le plus souvent courageux, qui se manifeste comme acte de résistance politique et comme conduite personnelle et sociale cohérente. On se rend compte que l'engagement de la plupart de ces acteurs s'enracine d'abord dans le «local», comme lieu d'authenticité, de réappropriation créatrice (selon l'expression de Jean Viard, 1994), d'innovation sociale et d'action. Le «local» se vit dans la solidarité de proximité : vivre ici, ensemble, entre nous. On y retrouve des éléments d'une "éthique du proche " (Baird Callicott, 1994), qui propose en cas de conflit éthique, un repère de responsabilité envers notre propre sphère de proximité (les êtres qui nous sont proches). On y retrouve aussi le land ethic (Aldo Leopold, 1949) ou l'éthique de la communauté biotique - ce tissu de vie dont la trame est faite de l'ensemble des êtres vivants et dont le fil est celui de la population humaine sur un territoire d'appartenance; une telle éthique inspire le biorégionalisme, selon lequel la culture et l'économie sont arrimées à l'écologie du bassin versant. Mais le local s'ouvre aussi sur un espace de solidarité élargie : «Vivre ensemble, sur Terre ${ }^{7}$, comme le propose l'éthique "gaïaniste» de James E. Lovelock (1986), qui met en évidence l'interdépendance des écosystèmes planétaires.

29 À travers le discours de nombreux acteurs des organisations œuvrant en éducation relative à l'éco-alimentation, nous avons saisi que l'engagement devient une charnière entre éthique et politique. L'engagement se fonde le plus souvent sur un système de valeurs caractérisé par un souci de l'autre, du collectif, par une préoccupation pour le respect et la promotion de l'intégrité de l'autre (autre humain et autre qu'humain) et pour le partage. S'ouvre ici, bien souvent, l'avenue politique de l'éthique de l'écologie sociale (Murray Bookchin, 1982, 2002), qu'elle soit explicite ou non. L'écologie sociale invite à repenser l'organisation du monde en dehors de la société capitaliste en raison de la contradiction entre la «pathologie » de la compétition du marché économique et la nature.

L'écologie sociale place l'« écologie " à un autre niveau de recherche et de praxis, bien au-delà d'un rapport de sollicitude, souvent romantique et mystique, avec une vague nature [...]. Elle se préoccupe de la relation très intime entre les humains et le monde organique autour d'eux. Elle donne à l'écologie une dimension révolutionnaire et politique. Nous devons travailler à opérer des changements non seulement dans le domaine de l'économie, mais dans les domaines subjectifs de la culture, de l'éthique, de l'esthétique [...]. (Bookchin, 2003)

L'éducation relative à l'éco-alimentation - qui, bien souvent, prend la forme d'une invitation à adopter certains choix de consommation ou à s'engager dans l'action sociale réflexive - participe au vaste mouvement du développement d'une population informée, consciente et critique, capable d'influence sur les politiques publiques, que ce soit sous forme de dénonciation, de résistance, d'innovations sociales ou de renforcement d'alternatives. Certes, on retrouve une diversité de fondements et de pratiques d'une telle éducation centrée sur les rapports entre alimentation et environnement, mais lorsqu'elle est abordée dans une perspective critique (celle de la critique sociale - Sauvé et Orellana, 2008), elle contribue à l'élaboration d'une argumentation à la fois éthique et politique pour des changements dans les modes de production, de distribution et de consommation agro-alimentaires, en lien avec un 
changement culturel majeur en ce qui a trait à notre rapport à l'environnement et aux questions socio-écologiques.

Le thème de l'alimentation offre ainsi à l'éducation relative à l'environnement un terrain particulièrement propice pour mettre en évidence le rapport étroit entre les systèmes de valeurs et les choix (ou non choix) politiques. La construction d'une éthique de l'environnement devient un acte politique, tout comme l'acte politique se réfère nécessairement à des fondements éthiques qu'il importe de clarifier. L'éducation - comme démarche de développement de compétences éthiques et de construction d'une « identité politique », et comme posture d'accompagnement et de valorisation de l'action sociale - devient ainsi elle-même un processus charnière entre éthique et politique.

\section{BIBLIOGRAPHIE}

BAPE - Bureau des audiences publiques sur l'environnement (2003). L'inscription de la production porcine dans le développement durable. Rapport d'enquête et d'audience publique. Québec : Bureau d'audiences publiques sur l'environnement.

Beauchamp, A. (1991). Pour une sagesse de l'environnement. Ottawa : Novalis.

Bookchin, M. (1982, 2002). The Ecology of Freedom - The Emergence and Dissolution of Hierarchy. New Hampshire : Silver Brook.

Bookchin, M. (2003). An overview of the roots of social ecology. Harbinger, A Journal of Social Ecology, 3(1). Consulté en janvier 2009 sur : http://www.social-ecology.org/harbinger/ vol3no1/ reflections.html

Callicott, B. (1994). Earth's Insights. A Multicultural Survey of Ecological Ethics from the Mediterranean Basin to the Australian Outback. Californie : University of California Press.

CSE - Conseil supérieur de l'éducation du Québec (1990). Développer une compétence éthique pour aujourd'hui : une tâche éducative essentielle. Rapport annuel 1989-1990 sur l'état et les besoins de l'éducation. Québec : Les Publications du Québec.

Des Jardins, J.R. (1995). Éthique de l'environnement. Une introduction à la philosophie de l'environnement. Sainte-Foy : Presses de l'Université du Québec.

Hansotte, M. (2005). Les intelligences citoyennes - Comment se prend et s'invente la parole collective. Bruxelles : De Boeck Université.

Heller, C. (2002). Désir, nature et société. L'écologie sociale au quotidien. Montréal : Éditions Écosociété.

Ladrière, J. (sans date). Engagement. Encyclopaedia universalis. Consulté en mars 2008 sur : http:// www.universalis.fr/encyclopedie

Larrère, C. (1997). Les philosophies de l'environnement. Paris : Presses Universitaires de France.

Leopold, A. (1949). A Sand County Almanach. New York : Oxford University Press. 
Lovelock, J. (1986). Gaia : the word as a living organism. New Scientist, 18, 25-28. Meadows, D., Meadows, D. et Randers, J. (2004). Limits to growth. The 30-year update. White River Junction : Chelsea green Publishing.

Pennac. D. (2007). Chagrins d'école. Paris : Gallimard.

Ricoeur, P. (1986). Du texte à l'action. Essais d'herméneutique II. Paris : Seuil.

Robin, M.-M. (2008). Le monde selon Monsanto. Montréal : Stanké.

Sauvé, L. et Orellana, I. (2008). Conjuguer rigueur, équité, créativité et amour : L'exigence de la criticité en éducation relative à l'environnement. (Texte éditorial). Éducation relative à

l'environnement : Regards - Recherches - Réflexions, 7, 7-23.

Sauvé, L. et Villemagne, C. (2003). L'éducation relative aux valeurs environnementales. Module 6. Programme d'études supérieures à distance - Formation en éducation relative à l'environnement - Francophonie internationale. Montréal : Les Publications ERE-UQAM, Université du Québec à Montréal - Collectif ERE-Francophonie.

Sauvé, L. et Villemagne, C. (2006). L'éthique de l'environnement comme projet de vie et « chantier » social : un défi de formation. Chemin de traverse - Revue transdisciplinaire en éducation à l'environnement, 2, 19-24.

Thomashow, M. (1995). Ecological Identity : Becoming a Reflective Environmentalist. Cambridge : MIT Press.

Viard, J. (1994). Les nouveaux enjeux du local. In Perrineau, P. (dir.), L'engagement politique. Déclin ou mutation? (p. 387-403). Paris : Presses de la Fondation nationale des sciences politiques.

\section{NOTES}

1. L'article suivant se penche plus en profondeur sur les défis d'une formation à l'« éducation relative aux valeurs environnementales » : Sauvé et Villemagne, 2006.

2. La notion d'intelligence citoyenne a été développée par Majo Hansotte (2005).

3. L'ouvrage suivant fait le tour de la question, sous ses aspects agronomiques, politiques, économiques, législatifs, sanitaires, écologiques et culturels : Proulx, D. et Sauvé, L. (2007). Porcheries! La porciculture intempestive au Québec. Montréal : Écosociété.

4. Une telle analyse mérite d'être faite aussi à propos du Rapport Agriculture et agroalimentaire: Assurer et bâtir l'avenir (2008), issu des travaux de la Commission sur l'avenir de l'agriculture et de l'agroalimentaire québécois au terme d'une vaste consultation publique en 2007. Les auteurs de ce rapport témoignent de deux visions du monde sous-jacentes au discours et non clarifiées comme telles: d'une part, la reconnaissance de la nécessité de repenser le système agroalimentaire au regard des problèmes socio-écologiques actuels (ce qui suppose un changement fondamental des modes de production, de distribution et de consommation) et d'autre part, paradoxalement, l'allégeance à l'économisme ambiant qui fait appel à une certaine "science ", à l'innovation et au développement technologique pour la création de "richesse ", amenant ainsi à tourner en rond pour la recherche de solutions, surtout sur le plan politique où il importerait d'effectuer des changements structurels. Une telle étude de cas est certes de nature à alimenter une formation à l'éthique de l'environnement.

5. Site Internet : http://www.unites.uqam.ca/ERE-UQAM

6. Site Internet : http://www.eco-alimentation.uqam.ca/

7. Il s'agit là du thème du $5^{\mathrm{e}}$ Congrès mondial d'éducation relative à l'environnement (Montréal, 2009, http://www.5weec.uqam.ca). 


\section{RÉSUMÉS}

L'un des objectifs majeurs d'une éducation relative à l'environnement axée sur l'approche critique des réalités socio-écologiques est certes de "déconstruire» la notion de "valeur environnementale» et les lieux communs du domaine de l'éthique, surtout lorsque c'est «la morale » qui tient lieu d'éthique. Un tel exercice amène à prendre conscience de la diversité des systèmes de valeurs et à reconnaître la dimension politique de ces constructions sociales. Cet article explore plus spécifiquement le lien entre l'éthique et le politique, et aborde certaines implications pédagogiques de la prise en compte de ce lien. Quelques éléments d'une recherche sur les théories et pratiques de l'éducation relative à l'éco-alimentation serviront à illustrer les propos.

One of the major goals of an environmental education centered on a critical approach of socioecological realities is to "deconstruct» the concept of «environmental value " and the commonplaces of the field of ethics, especially when it is "the moral » which takes place of ethics. Such an exercise makes us aware of the diversity of value systems and brings us to recognize the political dimension of these social constructs. More specifically, this article explores the link between ethics and politics, and presents some pedagogical implications of taking into account this link. Some elements of a research on the theories and practices of ecofood education will serve as examples.

\section{AUTEUR}

\section{LUCIE SAUVÉ}

Professeure titulaire au Département d'éducation et pédagogie de l'Université du Québec à Montréal (UQAM), Lucie Sauvé est également titulaire de la Chaire de recherche du Canada en éducation relative à l'environnement, membre de l'Institut des sciences de l'environnement et de l'Institut Santé et Société de l'UQAM. Elle est responsable du comité scientifique du Réseau international francophone de recherche en éducation relative à l'environnement (RefERE) et du Programme court d'études supérieures en éducation relative à l'environnement de l'UQAM. Elle est chercheure associée au Réseau québécois d'échange sur les questions autochtones (DIALOG). Ses principaux domaines de spécialisation ont trait à l'éducation relative à la santé environnementale, à l'éducation scientifique, à l'éducation à l'écodéveloppement, aux enjeux de la formation des éducateurs et à ceux de la participation communautaire en matière d'environnement. 